\title{
Suppression of NF-kB activation blocks osteoclastic bone resorption during estrogen deficiency
}

\author{
KAREN STRAIT ${ }^{1}$, YAN LI $^{3}$, DIRCK L. DILLEHAY ${ }^{1,2}$ and M. NEALE WEITZMANN ${ }^{3}$ \\ ${ }^{1}$ Division of Animal Resources, ${ }^{2}$ Department of Pathology and Laboratory Medicine and ${ }^{3}$ Division of Endocrinology, \\ Metabolism and Lipids, Department of Medicine, Emory University School of Medicine, Atlanta, GA 30322, USA
}

Received November 29, 2007; Accepted December 28, 2007

\begin{abstract}
Postmenopausal osteoporosis stems from an imbalance in osteoclastic bone resorption with respect to osteoblastic bone formation, a consequence of estrogen deficiency. The nuclear factor- $\mathrm{KB}(\mathrm{NF}-\mathrm{\kappa B})$ signal transduction pathway is critical for osteoclast formation and resorption, and suppression of NF- $\mathrm{kB}$ activation has been shown to block bone resorption in vitro, and to ameliorate inflammatory bone loss in vivo. The use of NF-кB antagonists to blunt the bone loss associated with estrogen deficiency however, has not been previously reported. In this study, we investigated whether pharmacological suppression of $\mathrm{NF}-\kappa \mathrm{B}$ signaling protects mice against ovariectomy (ovx)-induced bone loss. Ovx mice were treated with the potent $\mathrm{NF}-\kappa \mathrm{B}$ inhibitor pyrrolidine dithiocarbamate (PDTC) for 4 weeks and bone mineral density (BMD) and indices of bone structure quantitated by dual-energy X-ray absorptiometry (DXA), and $\mu$-computed tomography $(\mu \mathrm{CT})$. In vivo indices of bone resorption were quantitated in mouse serum using the biochemical marker C-terminal telopeptide of collagen (CTx). Our data revealed that $\mathrm{NF}-\mathrm{\kappa B}$ suppression significantly prevented ovx-induced bone destruction by preventing the increase in ovx-induced osteoclastic bone resorption. Our data suggest that $\mathrm{NF}-\kappa \mathrm{B}$ inhibitors may represent novel anticatabolic therapeutic agents for the amelioration of postmenopausal bone loss.
\end{abstract}

\section{Introduction}

Bone homeostasis is achieved by a delicate balance between osteoclastic bone resorption and osteoblastic bone formation. Numerous pathological processes have the capacity to disrupt

Correspondence to: Dr M. Neale Weitzmann, Division of Endocrinology, Metabolism and Lipids, 101 Woodruff Circle, WMRB 1305, Emory University School of Medicine, Atlanta, GA 30322, USA

E-mail:mweitzm@emory.edu

Key words: nuclear factor-кB, ovariectomy, osteoporosis, osteoclast this equilibrium leading to conditions where the rate of bone resorption outpaces the rate of bone formation leading to osteoporosis. Osteoporosis is a serious worldwide health threat. Postmenopausal osteoporosis is the archetypal osteoporotic condition in women after menopause and accounts for $71 \%$ of total fractures (1). The National Osteoporosis Foundation has estimated that one in two women over the age of 50 will have an osteoporosis-related fracture in their remaining lifetimes. Fractures incur monumental health care costs to patients and the community, and can have a serious negative impact on mobility and quality of life. Hip fractures almost always require surgery and the rate of mortality is considerable (2).

The NF- $\mathrm{NB}$ signal transduction pathway has long been recognized as critical for osteoclast development and function $(3,4)$ and double knockout of p50 and p52 NF-кB subunits leads to osteopetrosis due to a severe defect in osteoclast differentiation in these mice (5). Furthermore, suppression of NF-кB signaling in vitro blocks osteoclast activation (6). Recent studies have demonstrated the utility of pharmacological NF- $\mathrm{KB}$ suppression to inhibit osteoclast formation and activity associated with multiple myeloma in vitro (7) and in an animal model of rheumatoid arthritis in vivo (8).

Based on these data we hypothesize that pharmacological suppression of NF- $\mathrm{KB}$ in vivo may be an effective approach for ameliorating bone loss induced by estrogen deficiency by suppressing osteoclastic bone resorption.

PDTC is a well characterized NF- $\kappa \mathrm{B}$ inhibitor with demonstrated efficacy and good tolerability in numerous in vivo studies of inflammation (9-11). PDTC acts by stabilizing cytosolic IкB- $\alpha$ thus preventing the activation and nuclear translocation of the NF- $\kappa \mathrm{B}$ transcription factor (11).

In this study, we injected ovx mice with PDTC and investigated its impact on BMD, physical indices of bone structure using $\mu \mathrm{CT}$, and on in vivo biochemical markers of bone resorption (CTx). We show that $\mathrm{NF}-\kappa \mathrm{B}$ blockade by in vivo PDTC treatment significantly protects mice from bone loss following ovx. PDTC treatment alleviated bone loss by suppression of ovx-induced osteoclastic bone resorption. These data suggest that targeting of the NF-кB signal transduction pathway may constitute a novel therapeutic strategy for the amelioration of osteoporosis associated with numerous pathological conditions including postmenopausal osteoporosis. 


\section{Materials and methods}

Chemicals. Ammonium PDTC and all other reagents were purchased from the Sigma-Aldrich Chemical Corporation (St. Louis, MO), unless otherwise indicated.

Animals. All animal procedures were approved by the Emory University Animal Care and Use Committee and were used in accordance with the NIH Laboratory Guide for the Care and Use of Laboratory Animals.

Female C57BL6 mice 6 weeks of age were purchased from Charles River Laboratories (Raleigh, NC). Mice were acclimated for 4 weeks in the animal facility before use. Mice were housed in sterile polycarbonate cages with corn cob bedding on static racks and given gamma-irradiated 5V02 phytoestrogen-free mouse chow (Purina Mills, St. Louis, MO), and autoclaved water ad libitum. The animal facility was kept at $23 \pm 1^{\circ} \mathrm{C}$, with $50 \%$ relative humidity and a $12 / 12$ light/dark cycle.

Ovariectomy. Mice (10 weeks of age) were either shamoperated, or ovx. Ovx mice were divided into two groups receiving either vehicle (PBS) or PDTC at a dose of $80 \mathrm{mg} / \mathrm{kg}$ intraperitoneally 3 times per week.

Quantitation of BMD. In vivo BMD measurements of femurs (left and right femurs were averaged for each mouse) were made by DXA using a PIXImus2 bone densitometer (GE Medical Systems) as previously described (12).

$\mu C T . \mu \mathrm{CT}$ was performed using a $\mu \mathrm{CT} 40$ scanner, (Scanco Medical, Bassersdorf, Switzerland). Briefly, after careful dissection of muscle tissue, the right femur was fixed in $10 \%$ neutral-buffered formalin for $48 \mathrm{~h}$ and stored in $70 \%$ ethanol at $4^{\circ} \mathrm{C}$ until analysis. $\mu \mathrm{CT}$ analysis was performed by an operator blinded to the nature of the specimens. Bones were scanned at a resolution of $12 \mu \mathrm{m}$. For each sample 50 slices were taken at the identical starting position and covering a total area of $600 \mu \mathrm{m}$ proximal to the distal metaphyses. Static trabecular measurements were made using a cylindrical core sample that excluded cortical bone, with contouring for all subsequent slices. For visual representation one representative sample from each group was randomly selected for detailed three-dimensional (3D) reconstruction of core images from individual $\mu \mathrm{CT}$ slices.

Biochemical indices of bone resorption. CTx, a sensitive biochemical marker of bone resorption, was measured in mouse serum following an overnight fast, by 'RatLaps' a rodent-specific CTx ELISA (Nordic Bioscience Diagnostics A/S, Herlev, Denmark).

Statistical analysis. Statistical significance was determined using GraphPad InStat version 3 software for Windows XP (GraphPad Software, San Diego, CA). Multiple comparisons were performed by One-way ANOVA with Tukey-Kramer post test for parametric data, and Kruskal-Wallis post test for nonparametric data. $\mathrm{P} \leq 0.05$ was considered statistically significant. Groups were tested for normal distribution using the Kolmogorov-Smirnov test.

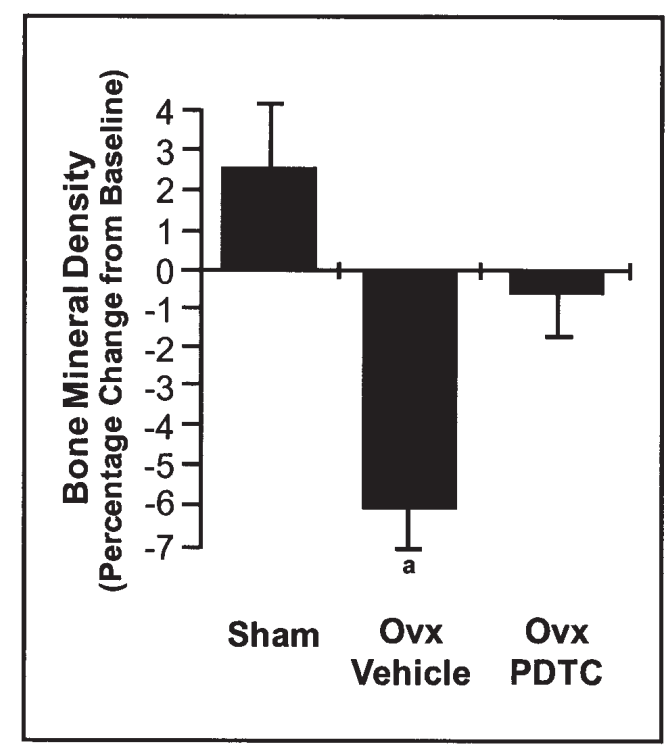

Figure 1. Suppression of $\mathrm{NF}-\kappa \mathrm{B}$ signaling by PDTC treatment in vivo protects mice from ovx-induced bone loss. BMD (bone mineral density) was quantitated by DXA 4 weeks after surgery in the femurs of sham-operated mice, ovx mice treated with vehicle (PBS), and ovx mice treated with the $\mathrm{NF}-\kappa \mathrm{B}$ antagonist PDTC ( $80 \mathrm{mg} / \mathrm{kg}$, IP, 3 times per week). BMD for left and right femurs were averaged for each mouse. $n=8-9$ mice/group. Data are presented as mean \pm SEM. ${ }^{a} \mathrm{P}<0.01$ vs. Sham; (One-way ANOVA; TukeyKramer post test).

\section{Results}

Pharmacological suppression of $N F-\kappa B$ signaling by PDTC treatment significantly reduces loss of BMD following ovx. BMD was quantitated by DXA at the femurs (average of left and right for each mouse) at baseline (time of surgery) and 4 weeks after surgery. While ovx led to a significant reduction in BMD, administration of PDTC significantly protected mice from ovx-induced bone loss at 4 weeks (Fig. 1).

Suppression of $N F-\kappa B$ signaling by PDTC treatment significantly reduces the decline in indices of bone structure measured by $\mu C T$, following ovx. We further verified the DXA findings using $\mu \mathrm{CT}$ analysis of femurs from each group of mice (Table I). The data demonstrate a significant 38\% decrease in trabecular bone volume per tissue volume (BV/TV) in vehicle-treated mice following ovx. This decrease resulted from reduced $\mathrm{BV}$ as TV was not significantly changed. Decreased BV resulted from a significant $14 \%$ decrease in trabecular thickness (Tb.Th.), $18 \%$ decrease in trabecular number (Tb.N.), and $43 \%$ decrease in trabecular connection density (Conn.D.), with a corresponding 22\% increase in trabecular separation (Tb.Sp.). Bone architecture denoted by the structure model index (SMI) was not significantly altered. Finally, volumetric trabecular volume BMD (TV.D) was decreased by $28 \%$ in ovx mice receiving vehicle. By contrast, ovx mice treated with PDTC were significantly protected from ovx-induced bone loss, and were not significantly different to sham groups. Protection was however observed to be incomplete and varied from $\sim 50$ to $70 \%$ protection depending on the index examined. 
Table I. $\mu \mathrm{CT}$ morphometric analysis of bone structure in sham-operated mice, ovx mice, and ovx mice receiving PDTC administration.

\begin{tabular}{|c|c|c|c|c|c|}
\hline Morphometric indices & Sham & Ovx + Vehicle & Ovx + PDTC & $\Delta$ Sham vs. Ovx & $\Delta$ Sham vs. Ovx + PDTC \\
\hline $\mathrm{TV}\left(\mathrm{mm}^{3}\right)$ & $1.6 \pm 0.1$ & $1.6 \pm 0.1$ & $1.70 \pm 0.1$ & 0.0 & 6.3 \\
\hline $\mathrm{BV}\left(\mathrm{mm}^{3}\right)$ & $0.10 \pm 0.01$ & $0.06 \pm 0.02^{\mathrm{a}}$ & $0.09 \pm 0.02^{\mathrm{b}}$ & -40.0 & -10.0 \\
\hline BV/TV (\%) & $6.0 \pm 0.5$ & $3.7 \pm 1.1^{\mathrm{a}}$ & $5.1 \pm 0.8^{b}$ & -38.3 & -15.0 \\
\hline Tb.Th. $(\mu \mathrm{m})$ & $45.5 \pm 4.6$ & $39.3 \pm 4.1^{\mathrm{a}}$ & $42.2 \pm 2.4$ & -13.6 & -7.3 \\
\hline Tb.N./mm & $3.3 \pm 0.2$ & $2.7 \pm 0.2^{\mathrm{a}}$ & $3.1 \pm 0.3$ & -18.2 & -6.1 \\
\hline $\mathrm{Tb} . \mathrm{Sp} .(\mu \mathrm{m})$ & $310.7 \pm 17.7$ & $377.7 \pm 25.3^{\mathrm{a}}$ & $337.8 \pm 34.2^{\mathrm{b}}$ & 21.6 & 8.7 \\
\hline SMI & $2.8 \pm 0.2$ & $2.8 \pm 0.3$ & $2.8 \pm 0.2$ & 0.0 & 0.0 \\
\hline Conn.D. $/ \mathrm{mm}^{3}$ & $62.6 \pm 13.4$ & $35.9 \pm 12.3^{\mathrm{a}}$ & $55.2 \pm 14.9^{\mathrm{b}}$ & -42.7 & -11.8 \\
\hline TV.D (mg HA/ $\left.\mathrm{cm}^{3}\right)$ & $134.9 \pm 18.4$ & $96.9 \pm 31.6^{\mathrm{a}}$ & $111.8 \pm 26.05$ & -28.2 & -17.1 \\
\hline
\end{tabular}

Trabecular indices of bone structure were computed from $\mu \mathrm{CT}$ scans. Data are presented as Mean \pm SD of 6 mice per group. Percentage change between Sham and Ovx + Vehicle and between Sham and Ovx + PDTC are shown in the last two columns. ${ }^{\text {a }} \leq 0.05$ vs. Sham; ${ }^{b} \mathrm{P} \leq 0.05$ vs. Ovx + Vehicle, One-way ANOVA with Tukey-Kramer post test: There were no statistically significant differences between Sham and Ovx + PDTC for any of the indices.

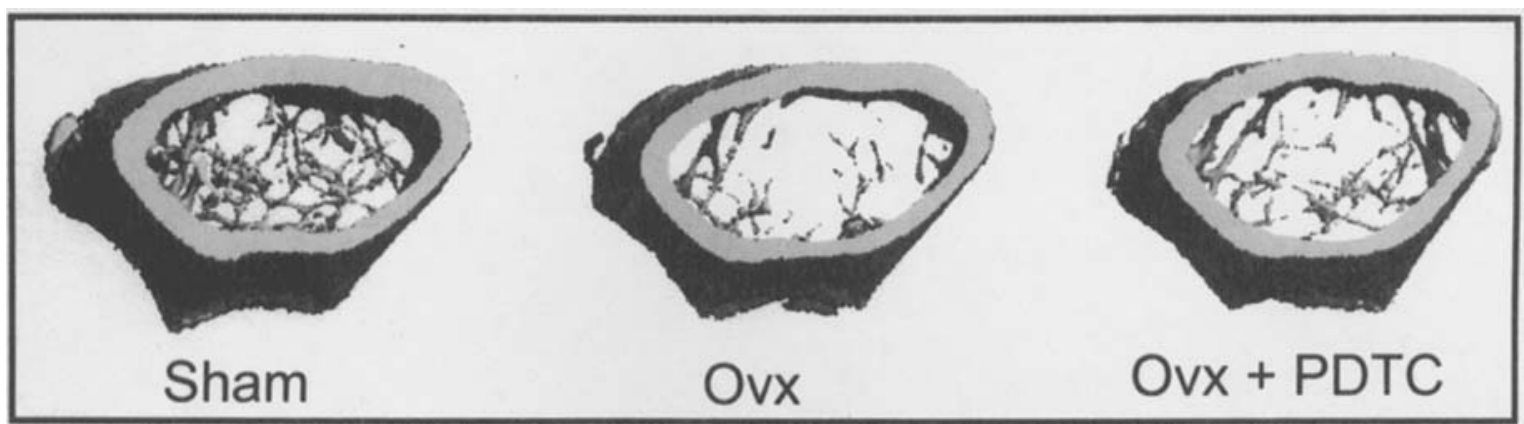

Figure 2. 3D $\mu \mathrm{CT}$ reconstruction of femurs. One representative femur from each group (sham-operated, ovx receiving vehicle and ovx receiving PDTC) was reconstructed from individual $\mu \mathrm{CT}$ scans at the distal metaphysis.

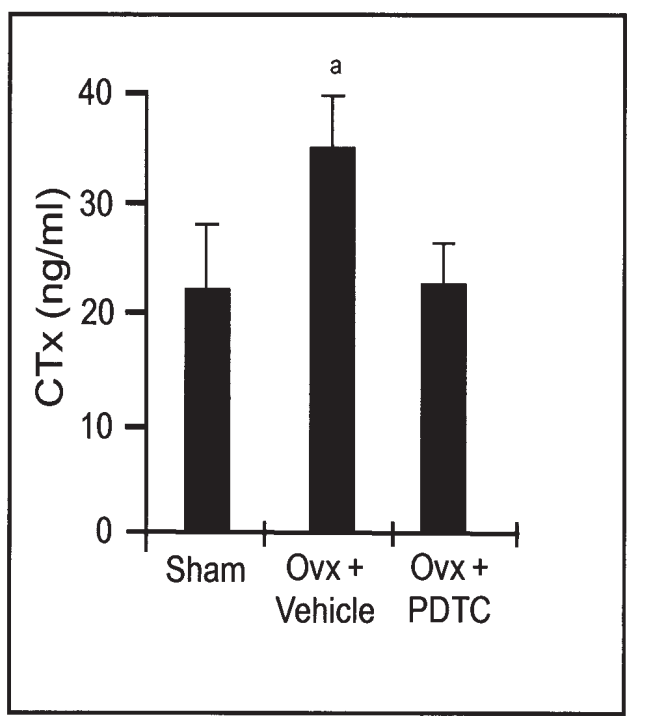

Figure 3. Biochemical markers of in vivo bone resorption. Four weeks after surgery CTx, a marker of bone resorption, was quantitated in the serum of each mouse. $\mathrm{n}=9-17$ mice/group. Data are presented as mean \pm SEM. ${ }^{*} \mathrm{P} \leq 0.05$; One-way ANOVA; Kruskal-Wallis post test.
Representative 3D reconstructions for one femur from each group selected at random is show for each group in Fig. 2.

Biochemical analysis of in vivo bone resorption. To quantitate indices of in vivo bone resorption we measured serum CTx, a specific and sensitive marker. The data revealed a significant $58 \%$ elevation in the rate of bone resorption induced by ovx (Fig. 3). PDTC treatment completely prevented the increase in bone resorption four weeks after the ovx.

\section{Discussion}

Our data demonstrate that suppression of $\mathrm{NF}-\kappa \mathrm{B}$ in vivo is an effective strategy for ameliorating osteoclastic bone breakdown under conditions of estrogen deficiency. Although PDTC treatment significantly reduced ovx-induced bone loss and PDTC-treated ovx mice were not statistically significantly different from sham mice for any of the $\mu \mathrm{CT}$ parameters, indices of bone structure nonetheless, fell short of completely returning to sham levels, falling typically between 50 and $70 \%$ protection. However, four weeks after PDTC treatment 
CTx levels indicated that bone resorption had completely returned to sham levels. These data suggest that the dose and/or frequency of administration of PDTC used in this particular experiment may have been inadequate to completely stall the initial wave of osteoclastic bone loss, but was ultimately effective in restoring osteoclast activity to sham levels by 4 weeks of treatment, thus preventing bone loss during the later stages of the study.

In addition, at the dose of PDTC used bone resorption returned to sham levels by 4 weeks of treatment, but did not completely abrogate bone resorption. Maintaining a basal rate of bone resorption is advantageous for remodeling of trabecular bone structure to achieve an optimal mechanical strength, and consequently the maintenance of a basal osteoclastic activity is desirable. It remains unknown as to whether PDTC administration in excess of 4 weeks would ultimately lead to a more pronounced suppression of osteoclast activity. At the relatively low dose of $80 \mathrm{mg} / \mathrm{kg}, 3$ times per week as used in this experiment, no ill effects were observed in the mice. A preliminary screening by a veterinary pathologist (D.L.D.) for toxic effects of PDTC breakdown in the liver and kidney, by examination of tissue sections stained with hematoxylin and eosin 4 weeks after ovx, revealed no evidence of PDTC-induced organ damage (data not shown). Nonetheless, we cannot exclude long-term toxic effects of PDTC breakdown products, or PDTC-induced toxicity or impaired function of other organs and tissues following longterm administration. Exposure to high doses of PDTC for extended periods (58 weeks) have been associated with myelin lesions and axonal degeneration, exacerbated by a high copper diet (13). Consequently, while PDTC is an effective agent for demonstrating a 'proof of principle' in animal models, longterm administration of NF- $\mathrm{KB}$ inhibitors in humans will likely require the development of agents with very low toxicity profiles and/or the direct targeting of $\mathrm{NF}-\kappa \mathrm{B}$ antagonists specifically to osteoclasts.

The molecular mechanisms by which NF-кB blockade ameliorates bone loss during estrogen deficiency are likely complex and multifactorial. $\mathrm{NF}-\kappa \mathrm{B}$ is a critical signal transduction pathway for several pro-osteoclastogenic cytokines including receptor activator of $N F-\kappa B$ Ligand (RANKL), as well as $\mathrm{TNF} \alpha$ and IL- 1 . TNF $\alpha$ directly synergizes with RANKL, the key osteoclastogenic effector, to amplify osteoclast formation (14-16). TNF $\alpha$ also directly stimulates osteoclast resorptive activity (17) and induces RANKL production by osteoblasts (18), potentially via induction of IL-1 $(18,19)$. IL-1 can also directly stimulate osteoclast precursor differentiation in the presence of permissive levels of RANKL (19).

From the osteoblast perspective we (20) and others (21-23) have reported that $\mathrm{TNF} \alpha$ is a potent suppressor of osteoblast differentiation and mineralization in vitro, and mediates its effects in part through NF- $\mathrm{KB}$ signaling that likely converges on multiple pathways including the suppression of Smads induced by early commitment factors such as TGF $\alpha$ and by pro-osteoblastic cytokines such as BMPs (20). PDTC may potentially eliminate this suppressive signal transmitted by $\mathrm{TNF} \alpha$, and may also act by directly stimulating osteoblast differentiation by blockading basal inhibitory NF-кB signals. Additional potential beneficial effects of NF- $\mathrm{KB}$ suppression on bone formation during ovx are thus possible and remain to be investigated.

The NF- $\kappa \mathrm{B}$ pathway is under intense investigation as a novel drug target for amelioration of numerous conditions including rheumatoid arthritis, Crohn's disease, infection, multiple myeloma, and myocardial infarction $(7,8,24)$. Our study suggests that estrogen deficiency-mediated bone loss may be another pathological condition that may ultimately benefit from NF- $\mathrm{KB}$ manipulation.

\section{Acknowledgements}

This work was supported by a grant from the University Research Committee of Emory University (M.N.W.). Y. Li is the recipient of T32 training award from the National Institute of Diabetes and Digestive and Kidney Diseases (DK007298). We thank Xiaoying Yang for excellent technical assistance with the $\mu \mathrm{CT}$.

\section{References}

1. Burge R, Dawson-Hughes B, Solomon DH, Wong JB, King A and Tosteson A: Incidence and economic burden of osteoporosisrelated fractures in the United States, 2005-2025. J Bone Miner Res 22: 465-475, 2007.

2. Lewis JR, Hassan SK, Wenn RT and Moran CG: Mortality and serum urea and electrolytes on admission for hip fracture patients. Injury 37: 698-704, 2006.

3. Franzoso G, Carlson L, Xing L, et al: Requirement for NFkappaB in osteoclast and B-cell development. Genes Dev 11: 3482-3496, 1997.

4. Boyce BF, Xing L, Franzoso G and Siebenlist U: Required and nonessential functions of nuclear factor-kappa B in bone cells. Bone 25: 137-139, 1999.

5. Iotsova V, Caamano J, Loy J, Yang Y, Lewin A and Bravo R: Osteopetrosis in mice lacking NF-kappaB1 and NF-kappaB2. Nat Med 3: 1285-1289, 1997.

6. Hall TJ, Schaeublin M, Jeker H, Fuller K and Chambers TJ: The role of reactive oxygen intermediates in osteoclastic bone resorption. Biochem Biophys Res Commun 207: 280-287, 1995.

7. Feng R, Anderson G, Xiao G, et al: SDX-308, a nonsteroidal anti-inflammatory agent, inhibits NF-kappaB activity, resulting in strong inhibition of osteoclast formation/activity and multiple myeloma cell growth. Blood 109: 2130-2138, 2007.

8. Dai S, Hirayama T, Abbas S and Abu-Amer Y: The IkappaB kinase (IKK) inhibitor, NEMO-binding domain peptide, blocks osteoclastogenesis and bone erosion in inflammatory arthritis. J Biol Chem 279: 37219-37222, 2004.

9. Carlson CG, Samadi A and Siegel A: Chronic treatment with agents that stabilize cytosolic IkappaB-alpha enhances survival and improves resting membrane potential in MDX muscle fibers subjected to chronic passive stretch. Neurobiol Dis 20: 719-730, 2005.

10. Jawien J, Gajda M, Mateuszuk L, et al: Inhibition of nuclear factor-kappaB attenuates artherosclerosis in apoE/LDLR double knockout mice. J Physiol Pharmacol 56: 483-489, 2005.

11. Cuzzocrea S, Chatterjee PK, Mazzon E, et al: Pyrrolidine dithiocarbamate attenuates the development of acute and chronic inflammation. Br J Pharmacol 135: 496-510, 2002.

12. Toraldo G, Roggia C, Qian WP, Pacifici R and Weitzmann MN: IL-7 induces bone loss in vivo by induction of receptor activator of nuclear factor kappa B ligand and tumor necrosis factor alpha from T cells. Proc Natl Acad Sci USA 100: 125-130, 2003.

13. Valentine HL, Amarnath K, Amarnath V and Valentine WM: Dietary copper enhances the peripheral myelinopathy produced by oral pyrrolidine dithiocarbamate. Toxicol Sci 89: 485-494, 2006.

14. Cenci S, Weitzmann MN, Roggia C, et al: Estrogen deficiency induces bone loss by enhancing T-cell production of TNF-alpha. J Clin Invest 106: 1229-1237, 2000.

15. Lam J, Takeshita S, Barker JE, Kanagawa O, Ross FP and Teitelbaum SL: TNF-alpha induces osteoclastogenesis by direct stimulation of macrophages exposed to permissive levels of RANK ligand. J Clin Invest 106: 1481-1488, 2000. 
16. Zhang YH, Heulsmann A, Tondravi MM, Mukherjee A and Abu-Amer Y: Tumor necrosis factor-alpha (TNF) stimulates RANKL-induced osteoclastogenesis via voupling of TNF type 1 receptor and RANK signaling pathways. J Biol Chem 276: 563-568, 2001.

17. Fuller K, Murphy C, Kirstein B, Fox SW and Chambers TJ: TNF-alpha potently activates osteoclasts, through a direct action independent of and strongly synergistic with RANKL. Endocrinology 143: 1108-1118, 2002.

18. Hofbauer LC, Lacey DL, Dunstan CR, Spelsberg TC, Riggs BL and Khosla S: Interleukin-1beta and tumor necrosis factoralpha, but not interleukin-6, stimulate osteoprotegerin ligand gene expression in human osteoblastic cells. Bone 25: 255-259, 1999.

19. Wei S, Kitaura H, Zhou P, Ross FP and Teitelbaum SL: IL-1 mediates TNF-induced osteoclastogenesis. J Clin Invest 115: 282-290, 2005.
20. Li Y, Li A, Strait K, Zhang H, Nanes MS and Weitzmann MN: Endogenous TNF-alpha lowers maximum peak bone mass and inhibits osteoblastic Smad activation, through NF-kappaB. J Bone Miner Res (In press).

21. Nanes MS: Tumor necrosis factor-alpha: molecular and cellular mechanisms in skeletal pathology. Gene 321: 1-15, 2003.

22. Gilbert LC, Rubin J and Nanes MS: The p55 TNF receptor mediates TNF inhibition of osteoblast differentiation independently of apoptosis. Am J Physiol Endocrinol Metab 288: E1011-E1018, 2005.

23. Lu X, Gilbert L, He X, Rubin J and Nanes MS: Transcriptional regulation of the Osterix (Osx, Sp7) promoter by tumor necrosis factor identifies disparate effects of mitogen-activated protein kinase and NFkappa-B pathways. J Biol Chem 281: 6297-6306, 2006.

24. Makarov SS: NF-kappaB as a therapeutic target in chronic inflammation: recent advances. Mol Med Today 6: 441-448, 2000 . 